\title{
THE SMOOTHED SPECTRAL ABSCISSA FOR ROBUST STABILITY OPTIMIZATION*
}

\author{
JORIS VANBIERVLIET ${ }^{\dagger}$, BART VANDEREYCKEN ${ }^{\dagger}$, WIM MICHIELS $^{\dagger}$, \\ STEFAN VANDEWALLE ${ }^{\dagger}$, AND MORITZ DIEHL ${ }^{\S}$
}

\begin{abstract}
This paper concerns the stability optimization of (parameterized) matrices $A(x)$, a problem typically arising in the design of fixed-order or fixed-structured feedback controllers. It is well known that the minimization of the spectral abscissa function $\alpha(A)$ gives rise to very difficult optimization problems, since $\alpha(A)$ is not everywhere differentiable, and even not everywhere Lipschitz. We therefore propose a new stability measure, namely the smoothed spectral abscissa $\tilde{\alpha}_{\epsilon}(A)$, which is based on the inversion of a relaxed $H_{2}$-type cost function. The regularization parameter $\epsilon$ allows to tune the degree of smoothness. For $\epsilon$ approaching zero, the smoothed spectral abscissa converges towards the nonsmooth spectral abscissa from above, so that $\tilde{\alpha}_{\epsilon}(A) \leq 0$ guarantees asymptotic stability. Evaluation of the smoothed spectral abscissa and its derivatives w.r.t. the matrix parameters $x$ can be performed at the cost of solving a primal-dual Lyapunov equation pair, allowing for an efficient integration into a derivative based optimization framework. Two optimization problems are considered: on the one hand the minimization of the smoothed spectral abscissa $\tilde{\alpha}_{\epsilon}(A(x))$ in function of the matrix parameters for a fixed value of $\epsilon$, and on the other hand the maximization of $\epsilon$ such that the stability requirement, $\tilde{\alpha}_{\epsilon}(A(x)) \leq 0$, is still satisfied. The latter problem can be interpreted as an $H_{2}$-norm minimization problem, and its solution additionally implies an upper bound on the corresponding $H_{\infty}$-norm, or a lower bound on the distance to instability. In both cases additional equality and inequality constraints on the variables can be naturally taken into account in the optimization problem.
\end{abstract}

AMS subject classifications. 93D09, 65K10, 49M20

Key words. robust stability, Lyapunov equations, eigenvalue optimization, pseudospectra

1. Introduction. Stability optimization of linear and nonlinear continuous-time dynamic systems is both a highly relevant and a difficult task. The optimization parameters often stem from a feedback controller, which can be used to optimize either a performance criterion or the asymptotic stability around a certain steady state. When also robustness against perturbations of the system must be taken into account, the resulting optimization problem becomes even more challenging.

Assuming an adequate parameterization of the desired feedback controller, the problem of finding a suitable steady state along with a stabilizing feedback controller can essentially be transformed into a nonlinear programming problem. By collecting all optimization variables in a vector $x$, we can summarize the described stability optimization problem as

$$
\min _{x} \Phi_{\text {stab }}(A(x)), \quad \text { s.t. } \quad g(x)=0, h(x) \leq 0,
$$

where $A(x)$ is the system matrix depending smoothly on $x$ and the function $\Phi_{\text {stab }}(\cdot)$

${ }^{*}$ This research was supported in part by the Research Council K.U.Leuven, CoE EF/05/006 Optimization in Engineering (OPTEC) and presents results of the Belgian Network DYSCO (Dynamical Systems, Control, and Optimization), funded by the Interuniversity Attraction Poles Programme, initiated by the Belgian State, Science Policy Office. The scientific responsibility rests with its authors. Bart Vandereycken is a Research Assistant of the Research Foundation - Flanders (FWO) and Wim Michiels is a Postdoctoral Fellow of the Research Foundation - Flanders (FWO).

${ }^{\dagger}$ Dept. of Computer Science, K.U.Leuven, Celestijnenlaan 200A, 3001 Leuven, Belgium.

E-mail: \{joris.vanbiervliet, bart.vandereycken, wim.michiels, stefan.vandewalle\}@cs.kuleuven.be

${ }^{\ddagger}$ Dept. of Mech. Engineering, T.U.Eindhoven, Den Dolech 2, 5612 Eindhoven, The Netherlands.

$\S$ Dept. of Electrical Engineering, K.U.Leuven, Kasteelpark Arenberg 10, 3001 Leuven, Belgium. E-mail: moritz.diehl@esat.kuleuven.be 
shall express our desire to optimize stability, under the given constraints. In the field of linear output feedback control, the closed-loop system matrix $A(x)$ will typically be of the form $A+B K C$, with $A$ the open-loop system matrix, $B$ and $C$ the input and output matrices, and $K$ containing the controller parameters $x$ to be optimized.

The most straightforward choice for the objective function $\Phi_{\text {stab }}$ is related to the eigenvalues of $A$, namely the spectral abscissa $\alpha(A)$. This value is defined as the real part of the rightmost eigenvalue of the spectrum $\Lambda(A)=\{z \in \mathbb{C} \mid \operatorname{det}(z I-A)=0\}$, that is, $\alpha(A):=\sup \{\Re(z) \mid z \in \Lambda(A)\}$.

The spectral abscissa is in general a non-Lipschitz and nonconvex function of $A$ $[13,14]$ and therefore typically a very hard function to optimize. Still, recent developments have led to algorithms that are able to tackle such nonsmooth objective functions $[8,12,22,23]$. The extension to infinite-dimensional systems has been made in [26]. Nonetheless, the spectral abscissa is also known to perform quite poorly in terms of robustness against parameter uncertainties. A tiny perturbation or disturbance to a parameter of a system that was optimized in the spectral abscissa can possibly lead to instability.

For this reason, more robust approaches have been proposed. Amongst those, the most prominent are $H_{\infty}$-optimization $[1,2,7,20,21]$ and, closely related, the minimization of the pseudospectral abscissa $[9,25]$. As these robust optimization formulations are connected to maximizing the distance to instability of the system under consideration, they inherently take the effect of perturbations into account in the stability measure. However, their objective functions still suffer from nonsmoothness and associated high computational costs in optimization. Throughout this paper, we will use the pseudo-spectral abscissa's standard notation $\alpha_{\epsilon}$, not to be confused with our symbol for the smoothed spectral abscissa, namely $\tilde{\alpha}_{\epsilon}$. Another, albeit less widespread, robustness measure is the robust spectral abscissa, denoted by $\alpha_{\delta}$ as in [8], and is based on Lyapunov variables.

The paper is organized as follows. In $\S 2$, we define the smoothed spectral abscissa and we outline its most important properties. Section 3 discusses how to efficiently compute this newly defined stability measure along with its derivatives. In $\S 4$, we explain how the smoothed spectral abscissa can be used to formulate optimization problems dealing with robust stability and $\S 5$ draws a relation with the pseudo-spectral abscissa. Finally, we illustrate our stabilization method by treating two numerical examples in $\S 6$.

2. The smoothed spectral abscissa. In this section we introduce a new stability measure, namely the smoothed spectral abscissa. In addition to alleviating the problem of nonsmoothness, it also has certain beneficial robustness properties. At its basis is the following well-known stability criterion.

Lemma 2.1. For any matrix norm $\|\cdot\|$ the matrix $A \in \mathbb{R}^{n \times n}$ is Hurwitz stable if and only if the integral $\int_{0}^{\infty}\|\exp (A t)\|^{2} \mathrm{~d} t$ is finite.

Inspired by this observation, we introduce the matrix function $f: \mathbb{R}^{n \times n} \times \mathbb{R} \cup$ $\{\infty\} \rightarrow \mathbb{R} \cup\{\infty\}$, that uses the Frobenius norm $\|M\|_{\mathrm{F}}^{2}:=\operatorname{trace}\left(M^{\mathrm{T}} M\right)$ and takes as its arguments, next to the matrix $A$, also a real-valued relaxation parameter $s$

$$
f(A, s):=\int_{0}^{\infty}\left\|V \mathrm{e}^{(A-s I) t} U\right\|_{\mathrm{F}}^{2} \mathrm{~d} t .
$$

Here, the matrices $U$ and $V$ are to be seen as respective input and output weighting matrices, with $(A, U)$ controllable and $(V, A)$ observable. It is easy to see that $f(A, s)$ is nothing else than the squared weighted and relaxed $H_{2}$-norm of a system with 
transfer function $\mathbf{H}_{s}(z)=V(z I-(A-s I))^{-1} U$, i.e.,

$$
f(A, s)=\left\|\mathbf{H}_{s}\right\|_{\mathcal{H}_{2}}^{2} .
$$

We continue with the following properties for the function $f(A, s)$.

Lemma 2.2. $\forall A \in \mathbb{R}^{n \times n}:\{f(A, s) \mid s>\alpha(A)\}=\mathbb{R}_{0}^{+}$.

Proof. If $s>\alpha(A)$, the matrix $A-s I$ is stable and therefore $f(A, s)$ is finite by Lemma 2.1. Additionally, $f(A, s)$ tends to infinity and to zero for $s \rightarrow \alpha(A)$ and $s \rightarrow \infty$ respectively.

Lemma 2.3. $\forall s>\alpha(A): \partial f(A, s) / \partial s<0$ and $\partial^{2} f(A, s) / \partial s^{2}>0$.

Proof. This can be verified by differentiating the integral in equation (2.1) to $s$ once and twice respectively.

These last two properties allow us to introduce the implicit function of the relation $f(A, s)=\epsilon^{-1}$ w.r.t. the relaxation argument $s$, as it is well defined on the whole domain, that is, for any $\epsilon>0$ and for any matrix $A \in \mathbb{R}^{n \times n}$. We will call this function the "smoothed spectral abscissa", analogously to the smoothed spectral radius for discrete time systems [15].

DeFinition 2.4. The smoothed spectral abscissa is the map $\alpha: \mathbb{R}_{0}^{+} \times \mathbb{R}^{n \times n} \rightarrow \mathbb{R}$ : $(\epsilon, A) \mapsto \tilde{\alpha}_{\epsilon}(A)$ that uniquely solves

$$
f\left(A, \tilde{\alpha}_{\epsilon}(A)\right)=\epsilon^{-1} .
$$

Because $f(A, s)$ is analytic in both its arguments for any $s>\alpha(A)$, it follows from the implicit function theorem that $\tilde{\alpha}_{\epsilon}(A)$ is analytic on its whole domain $\epsilon>0$, $A \in \mathbb{R}^{n \times n}$. Moreover, it has the following additional properties.

TheOREM 2.5. $\tilde{\alpha}_{\epsilon}(A)$ is an increasing function of $\epsilon$, that is, $\partial \tilde{\alpha}_{\epsilon}(A) / \partial \epsilon>0$.

Proof. Differentiating (2.3) on both sides w.r.t. $\epsilon$, we obtain

$$
\frac{\mathrm{d} f\left(A, \tilde{\alpha}_{\epsilon}(A)\right)}{\mathrm{d} \epsilon}=\frac{\partial f(A, s)}{\partial s} \frac{\partial \tilde{\alpha}_{\epsilon}(A)}{\partial \epsilon}=-\epsilon^{-2}<0,
$$

from which the proposition holds by Lemma 2.3 .

TheOREM 2.6. $\forall \epsilon>0: \tilde{\alpha}_{\epsilon}(A)>\alpha(A)$ and $\lim _{\epsilon \rightarrow 0} \tilde{\alpha}_{\epsilon}(A)=\alpha(A)$.

Proof. These two properties follow from the fact that $f(A, s)$ is finite and descending for $s>\alpha(A)$, but tends to infinity as $s$ approaches $\alpha(A)$.

Also note that this last theorem implies that a nonpositive smoothed spectral abscissa guarantees that the underlying system is asymptotically stable. The above definition and properties are illustrated in Figure 2.1.

3. Computing the smoothed spectral abscissa and its derivatives. Having defined the smoothed spectral abscissa, we now take a look at its computation. As explained in the previous section, this involves solving the smooth but nonlinear equation $f(A, s)=\epsilon^{-1}$ for $s$. Therefore, we first give some properties of the function $f(A, s)$ regarding its evaluation and its derivatives.

Lemma 3.1. For all $s>\alpha(A)$, there exist symmetric $n \times n$ matrices $P$ and $Q$ such that

$$
\begin{aligned}
f(A, s) & =\operatorname{trace}\left(V P V^{\mathrm{T}}\right)=\operatorname{trace}\left(U^{\mathrm{T}} Q U\right), \\
\frac{\partial f(A, s)}{\partial s} & =-2 \operatorname{trace}(Q P)=-2 \operatorname{trace}(P Q), \\
\frac{\partial f(A, s)}{\partial A} & =2 Q P
\end{aligned}
$$




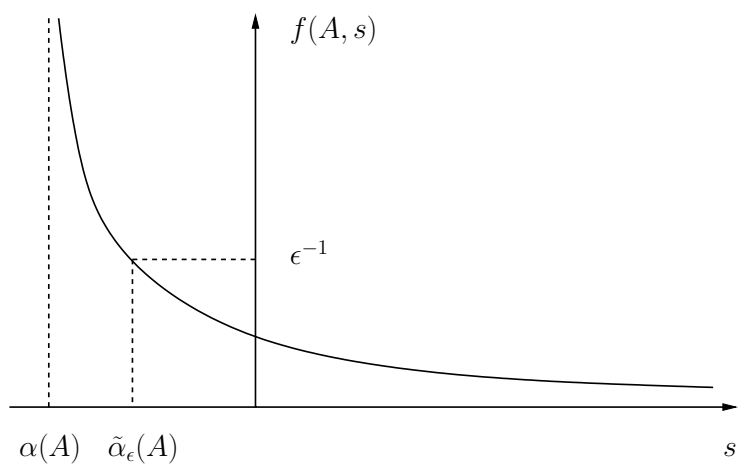

FIG. 2.1. Typical behaviour of the function $f(A, s)$ in function of $s$. The smoothed spectral abscissa $\tilde{\alpha}_{\epsilon}(A)$ is defined as the abscissa of the point where this function reaches $\epsilon^{-1}$.

where $P$ and $Q$ satisfy the primal-dual Lyapunov equation pair

$$
\begin{aligned}
& 0=L(P, A, U, s), \\
& 0=L\left(Q, A^{\mathrm{T}}, V^{\mathrm{T}}, s\right),
\end{aligned}
$$

with $L$ defined as

$$
L(P, A, U, s):=(A-s I) P+P(A-s I)^{\mathrm{T}}+U U^{\mathrm{T}} .
$$

Proof. The first part follows immediately by writing out the Frobenius norm in $(2.1)$

$$
f(A, s)=\operatorname{trace}\left(V \int_{0}^{\infty} \mathrm{e}^{(A-s I) t} U U^{\mathrm{T}} \mathrm{e}^{(A-s I)^{\mathrm{T}} t} \mathrm{~d} t V^{\mathrm{T}}\right)
$$

and by the well-known fact that, since $A-s I$ is stable, the above integral can be identified as the trace of $P$, the solution of equation (3.2a) (see, for instance, $[17,27]$ ). Note that solving the dual Lyapunov equation (3.2b) computes a matrix $Q$ that solves the dual integral

$$
Q=\int_{0}^{\infty} \mathrm{e}^{(A-s I)^{\mathrm{T}} t} V^{\mathrm{T}} V \mathrm{e}^{(A-s I) t} \mathrm{~d} t
$$

Since $A$ is fixed in the partial derivative $\frac{\partial f(A, s)}{\partial s}$, we can regard $f$ as a function of $P$, where $P$ depends on $s$ through the Lyapunov relation $L(P(s), A, U, s)$. Rather than computing this partial derivative directly as

$$
\frac{\partial f(A, s)}{\partial s}=\frac{\mathrm{d}}{\mathrm{d} s} \operatorname{trace}\left(V P(s) V^{\mathrm{T}}\right)=\operatorname{trace}\left(V \frac{\mathrm{d} P}{\mathrm{~d} s} V^{\mathrm{T}}\right)
$$

with $\frac{\mathrm{d} P}{\mathrm{~d} s}$ the solution of the Lyapunov equation $(A-s I) \frac{\mathrm{d} P}{\mathrm{~d} s}+\frac{\mathrm{d} P}{\mathrm{~d} s}(A-s I)^{\mathrm{T}}-2 P=0$, we choose to use an adjoint differentiation technique. Vectorizing the matrix $P$ in a $n^{2} \times 1$ vector $p=\operatorname{vec}(P)$, we can write

$$
\frac{\partial f}{\partial s}=\frac{\partial f}{\partial p} \frac{\partial p}{\partial s}=-\frac{\partial f}{\partial p}\left(\frac{\partial \ell}{\partial p}\right)^{-1} \frac{\partial \ell}{\partial s},
$$


where $\ell:=\operatorname{vec}(L(P, A, U, s))$ represents the vectorized primal Lyapunov equation (3.2a). Making use of the fact that $\operatorname{vec}\left(M X N^{T}\right)=(N \otimes M) \operatorname{vec}(X)$ [16], we can make $\ell$ explicit in $p$, obtaining the following $n^{2} \times n^{2}$ linear system.

$$
\ell(p, A, U, s)=\frac{\partial \ell}{\partial p} p+\operatorname{vec}\left(U U^{\mathrm{T}}\right)=0,
$$

with $\frac{\partial \ell}{\partial p}=(A-s I) \otimes I+I \otimes(A-s I)$ and $\otimes$ denoting the Kronecker product. Similarly for the dual Lyapunov equation, we get

$$
\ell\left(q, A^{\mathrm{T}}, V^{\mathrm{T}}, s\right)=\frac{\partial \ell}{\partial q} q+\operatorname{vec}\left(V^{\mathrm{T}} V\right)=0, \text { with } \frac{\partial \ell}{\partial q}=(A-s I)^{\mathrm{T}} \otimes I+I \otimes(A-s I)^{\mathrm{T}} .
$$

It is easily verified that $\frac{\partial \ell}{\partial q}=\frac{\partial \ell^{\mathrm{T}}}{\partial p}$. Replacing $\frac{\partial \ell}{\partial q}$ in the relation $\ell\left(q, A^{\mathrm{T}}, V^{\mathrm{T}}, s\right)=0$, and using in addition the fact that $\operatorname{vec}\left(V^{\mathrm{T}} V\right)$ equals $\frac{\partial f^{\mathrm{T}}}{\partial p}$, we find that

$$
\frac{\partial \ell^{\mathrm{T}}}{\partial p} q+\frac{\partial f^{\mathrm{T}}}{\partial p}=0 \quad \Leftrightarrow \quad q^{\mathrm{T}}=-\frac{\partial f}{\partial p}\left(\frac{\partial \ell}{\partial p}\right)^{-1} .
$$

Substituting into (3.3), along with $\frac{\partial \ell}{\partial s}=-2 p$, finally gives

$$
\frac{\partial f}{\partial s}=q^{\mathrm{T}}(-2 p)=-2 \operatorname{vec}(Q)^{\mathrm{T}} \operatorname{vec}(P)=-2 \operatorname{trace}(Q P) .
$$

For the third part of the proof, i.e. the proof of the expression for the derivative w.r.t. $A$, we can use the same adjoint differentiation technique. Hereto, we again let $f$ depend on the vectorized matrix $p=\operatorname{vec}(P)$, which now depends on $a=\operatorname{vec}(A)$ according to the relation $\ell(p(a), a, s)=0$. Using the previous results, we obtain

$$
\operatorname{vec}^{\mathrm{T}}\left(\frac{\partial f}{\partial A}\right)=\frac{\partial f}{\partial a}=\frac{\partial f}{\partial p} \frac{\partial p}{\partial a}=-\frac{\partial f}{\partial p}\left(\frac{\partial \ell}{\partial p}\right)^{-1} \frac{\partial \ell}{\partial a}=q^{\mathrm{T}} \frac{\partial \ell}{\partial a} .
$$

To find $\frac{\partial \ell}{\partial a}$, we first have to make $\ell$ explicit in $a$, which yields

$$
\ell(P, a, U, s)=\frac{\partial \ell}{\partial a} a+\operatorname{vec}\left(U U^{\mathrm{T}}\right)=0 \quad \text { with } \quad \frac{\partial \ell}{\partial a}=(P \otimes I)+(I \otimes P) \Pi,
$$

where $\Pi$ denotes the symmetric permutation matrix that satisfies $\operatorname{vec}\left(A^{\mathrm{T}}\right)=\Pi \operatorname{vec}(A)$. Filling in in (3.4) gives

$$
\operatorname{vec}^{\mathrm{T}}\left(\frac{\partial f}{\partial A}\right)=\left(\frac{\partial \ell^{\mathrm{T}}}{\partial a} q\right)^{\mathrm{T}}=\left[\operatorname{vec}(Q P)+\Pi^{\mathrm{T}} \operatorname{vec}(P Q)\right]^{\mathrm{T}}=2 \operatorname{vec}^{\mathrm{T}}(Q P)=\operatorname{vec}^{\mathrm{T}}(2 Q P) .
$$

By comparison of both sides, we finally obtain that

$$
\frac{\partial f}{\partial A}=2 Q P
$$

which concludes the proof.

The relatively cheap computation of $f(A, s)$ and its derivative w.r.t. $s$ enables us to efficiently solve the nonlinear equation $f(A, s)=\epsilon^{-1}$ by use of standard root finding methods, and thus evaluate the smoothed spectral abscissa $\tilde{\alpha}_{\epsilon}(A)$. Specifically, we can use a Dekker-Brent type method [6] provided that we establish a root 
bracketing interval first, or Newton's method if we want to exploit the availability of the derivatives. For further elaboration on the computational issues involving the smoothed spectral abscissa, we refer to $\S 6.3$

As we will want to use derivative-based optimization methods later on to exploit the smoothness of the smoothed spectral abscissa, we need to be able to compute also the derivative of $\tilde{\alpha}_{\epsilon}(A)$ w.r.t. $A$. Fortunately, this can be done at almost no extra cost. Indeed, the same ingredients that were needed for the evaluation of $\tilde{\alpha}_{\epsilon}(A)$, namely the solutions $P$ and $Q$ of one primal-dual Lyapunov equation pair, give us direct access to the derivative of $\tilde{\alpha}_{\epsilon}(A)$ w.r.t. $A$, as expressed in the following theorem.

TheOREM 3.2. For fixed $\epsilon$, the derivative of the smoothed spectral abscissa $\tilde{\alpha}_{\epsilon}(A)$ w.r.t. A equals

$$
\frac{\partial \tilde{\alpha}_{\epsilon}(A)}{\partial A}=\frac{Q P}{\operatorname{trace}(Q P)},
$$

where $P$ and $Q$ satisfy the Lyapunov equation pair (3.2a) - (3.2b) for $s=\tilde{\alpha}_{\epsilon}(A)$.

Proof. Differentiating the implicit equation $f(A, s)=\epsilon^{-1}$ w.r.t. $A$, and using the chain rule, we obtain

$$
\frac{\partial \tilde{\alpha}_{\epsilon}(A)}{\partial A}=-\left(\frac{\partial f(A, s)}{\partial s}\right)^{-1}\left(\frac{\partial f(A, s)}{\partial A}\right) .
$$

Recalling (3.1b) and (3.1c) of Lemma 3.1, the result follows directly.

Remark 1. Suppose $A$ depends on an $m \times 1$ parameter vector $x$, then a direct approach to compute the derivatives w.r.t. to these parameters would require solving $m+1$ Lyapunov equations with different right-hand sides, instead of $m+1$ matrix multiplications of $\partial A / \partial x$ with $\partial \tilde{\alpha}_{\epsilon} / \partial A$.

4. Robust stability optimization. When it comes to algorithmic optimization, a first major advantage of the smoothed spectral abscissa criterion is that it is differentiable everywhere, and that its derivatives can be computed efficiently. This allows us to use derivative based methods without any restriction. Additionally, due to its differentiable dependence on $A$ and its connection with the $H_{2}$-norm, it is expected to be a more robust measure for stability than the spectral abscissa. We will present two smooth formulations of the stability optimization problem (1.1), one that focuses on mere stabilization, and one that will turn out to perform a $H_{2}$-norm minimization.

The first variant is to simply choose a fixed $\epsilon>0$ and then solve

$$
\min _{x} \tilde{\alpha}_{\epsilon}(A(x)), \quad \text { s.t. } \quad g(x)=0, h(x) \leq 0 .
$$

Here, $\tilde{\alpha}_{\epsilon}(A(x))$ is indirectly dependent on the matrix parameter vector $x$, as it is implicitly defined as the solution of the relation $f(A(x), s)=\epsilon^{-1}$ w.r.t. $s$. By decoupling this implicit relation into a constraint, we can formulate the problem alternatively as

$$
\min _{x} s, \quad \text { s.t. } \quad f(A(x), s)=\epsilon^{-1}, g(x)=0, h(x) \leq 0,
$$

which is more amenable for an SQP optimization framework.

Should problem (4.1) or (4.2) not result in a negative optimum for the chosen $\epsilon$, then one can try again with a smaller $\epsilon$. Note also that, if the sole goal is to achieve a stable system, one may break the optimization procedure once the smoothed spectral abscissa becomes smaller than zero. 
In the minimization formulation of the smoothed spectral abscissa with fixed $\epsilon$, the choice of $\epsilon$ is somewhat arbitrary. As indicated by Theorem 2.6, $\tilde{\alpha}_{\epsilon}(A)$ becomes smoother - and thus presumably a more robust measure for stability - with increasing values for $\epsilon>0$. Thus, we might alternatively search for the largest $\epsilon$ so that the stability certificate $\tilde{\alpha}_{\epsilon}(A) \leq 0$ still holds. This leads to a second optimization problem

$$
\max _{x, \epsilon} \epsilon \quad \text { s.t. } \quad \tilde{\alpha}_{\epsilon}(A(x)) \leq 0 \quad \text { and } \quad g(x)=0, h(x) \leq 0 .
$$

Since $\tilde{\alpha}_{\epsilon}(A)$ is a continuously growing function of $\epsilon$, the constraint in problem (4.3) will always be active in its optimum $\left(x^{*}, \epsilon^{*}\right)$. Hence, it is easily seen that the solution of the first problem (4.1), with $\epsilon$ fixed to $\epsilon^{*}$, will be exactly zero, and that in addition its minimizer will be the same as the one for problem (4.3), namely $x^{*}$. Succinctly,

$$
x^{*}=\arg \min _{x} \tilde{\alpha}_{\epsilon^{*}}(A(x)) \quad \text { and } \quad \tilde{\alpha}_{\epsilon^{*}}\left(A\left(x^{*}\right)\right)=0 .
$$

Problem (4.3) can thus be solved by finding the $\epsilon$ for which the resulting minimal smoothed spectral abscissa is zero, which can be implemented by bisecting $\epsilon$. The activeness of the stability constraint also leads to the following nice interpretation of problem (4.3).

TheOREM 4.1. Any solution $x^{*}$ that solves problem (4.3) also solves the $\mathrm{H}_{2}$-norm optimization of a system with transfer function $\mathbf{H}(x)(z):=V(z I-A(x))^{-1} U$, i.e.

$$
x^{*}=\arg \min _{x}\|\mathbf{H}(x)\|_{\mathcal{H}_{2}}, \quad \text { s.t. } \quad g(x)=0, h(x) \leq 0,
$$

and the solution $\left\|\mathbf{H}\left(x^{*}\right)\right\|_{\mathcal{H}_{2}}$ is equal to $\sqrt{1 / \epsilon^{*}}$.

Proof. Taking the inverse of the objective function in problem (4.3) and incorporating the activeness of the stability constraint, this problem rewrites as the minimization of the function $f(A(x), 0)$, subject to the constraints, and additionally restricting $x$ to values for which $A(x)$ is stable. This problem is, by (2.2), equivalent to minimizing the (squared) $\mathrm{H}_{2}$-norm of the system with transfer function $\mathbf{H}$.

REMARK 2. Solving problem (4.3) with the restriction $\tilde{\alpha}_{\epsilon}<s$ (with $s<0$ ) would minimize the $\mathrm{H}_{2}$-norm of a system with the shifted transfer function $\mathbf{H}_{s}$.

5. Relation with the pseudo-spectral abscissa. We will now draw a relationship between the smoothed spectral abscissa $\tilde{\alpha}_{\epsilon}(A)$ and the pseudo-spectral abscissa $\alpha_{\epsilon}(A)$, the latter being defined as

$$
\alpha_{\epsilon}(A):=\sup \left\{\Re(z) \mid z \in \Lambda(X) \text { and }\|X-A\|_{2} \leq \epsilon\right\} .
$$

Recall the following well-known equivalency involving $\alpha_{\epsilon}(A)$ and the corresponding $\mathcal{H}_{\infty}$-norm [9]

$$
\alpha_{\epsilon}(A)<0 \Leftrightarrow \max _{\Re(z)=0}\left\|(z I-A)^{-1}\right\|_{2}=\|\mathbf{H}\|_{\mathcal{H}_{\infty}}<\epsilon^{-1}
$$

We can also interpret this in terms of the $\mathcal{H}_{\infty}$-norm of a shifted matrix $A-s I$. We then get

$$
\alpha_{\epsilon}(A-s I)<0 \quad \Leftrightarrow \quad \alpha_{\epsilon}(A)<s \quad \Leftrightarrow \quad\left\|\mathbf{H}_{s}\right\|_{\mathcal{H}_{\infty}}<\epsilon^{-1} .
$$

In other words, the pseudo-spectral abscissa is the minimal shift-to-the-left $s$ for which the "shifted" $\mathcal{H}_{\infty}$-norm is smaller than $\epsilon^{-1}$. 
Going back to the definition of the smoothed spectral abscissa $\tilde{\alpha}_{\epsilon}(A)$, and taking into account that $f$ is a decreasing function of $s$ (Lemma 2.3), we derive a similar relation as we did in (5.2)

$$
\tilde{\alpha}_{\epsilon}(A)<s \quad \Leftrightarrow \quad f(A, s)=\left\|\mathbf{H}_{s}\right\|_{\mathcal{H}_{2}}^{2}<\epsilon^{-1} .
$$

Analogously, we can regard the smoothed spectral abscissa as the minimal shift $s$ for which $\left\|\mathbf{H}_{s}\right\|_{\mathcal{H}_{2}}^{2}$ lies below the bound $\epsilon^{-1}$ (see also Figure 2.1). Thus, $\alpha_{\epsilon}(A)$ and $\tilde{\alpha}_{\epsilon}(A)$ are both relaxations of the spectral abscissa in the sense that they are both induced by placing a bound on a norm $\left(\mathcal{H}_{\infty}\right.$ and $\mathcal{H}_{2}$ respectively) that goes to infinity when approaching instability. This analogy enables us to relate these two robust stability measures.

TheOrem 5.1 (Relation to pseudo-spectral abscissa). For $s>\alpha(A)$ and for $U=V=I$, the following holds

$$
\begin{aligned}
\left\|\mathbf{H}_{s}\right\|_{\mathcal{H}_{\infty}} & <2\left\|\mathbf{H}_{s}\right\|_{\mathcal{H}_{2}}^{2} \\
\alpha_{\epsilon / 2}(A) & <\tilde{\alpha}_{\epsilon}(A) .
\end{aligned}
$$

Proof. The first inequality is based on [3], where $2 \lambda_{\max }\left(Q^{2}\right)^{\frac{1}{2}}$ is established to be an upper bound on the $H_{\infty}$-norm of an unweighted system with transfer function $\mathbf{H}_{s}$, where $Q$ satisfies $(3.2 \mathrm{~b})$. Since $Q$ is a positive definite matrix, we can deduce from this the following.

$$
\left\|\mathbf{H}_{s}\right\|_{\mathcal{H}_{\infty}} \leq 2 \lambda_{\max }(Q)<2 \operatorname{trace}(Q)
$$

This proves (5.4a) directly by Lemma 3.1(a) and by (2.2). Suppose then, by (5.3), that for $s=\tilde{\alpha}_{\epsilon}(A)$ it is true that $\left\|\mathbf{H}_{s}\right\|_{\mathcal{H}_{2}}^{2}=\epsilon^{-1}$. Using (5.4a) in connection with (5.2), assertion (5.4b) follows.

This property has an important implication in terms of robust optimization. It shows that the squared $H_{2}$-norm constitutes an upper bound to the $H_{\infty}$-norm, which is directly related to the distance to instability of a system. By minimizing the first norm, one could expect that the second norm should also go down.

On top of this rather intuitive result, $(5.4 \mathrm{~b})$ also provides guarantees that come with a negative smoothed spectral abscissa. Indeed, if $\tilde{\alpha}_{\epsilon}(A(x))<0$ for some $x$, we are not only sure that the system with system matrix $A(x)$ will be a stable one, but also that this system will have a $H_{\infty}$-norm smaller than $2 / \epsilon$. In other words, we are certain that the distance to instability of the system will be at least $\epsilon / 2$.

6. Numerical examples. We will now put theory into practice by treating two control examples using the smoothed spectral abscissa as the stability criterion. First, we will illustrate the theory behind the smoothed spectral abscissa by use of an academic example. Next, we will treat a more realistic example, namely a turbo generator model. We conclude by making a comparison of the computational cost of the smoothed spectral abscissa in relation with other robust stability measures. All computations were done with MATLAB R2008a.

6.1. A simple state feedback controlled system. Consider the following two-parameter linear state feedback controlled system, with a closed-loop system matrix $A+B K$, and where

$$
A=\left[\begin{array}{ccc}
0.1 & -0.03 & 0.2 \\
0.2 & 0.05 & 0.01 \\
-0.06 & 0.2 & 0.07
\end{array}\right], \quad B=\frac{1}{2}\left[\begin{array}{c}
-1 \\
-2 \\
1
\end{array}\right], \quad K^{\mathrm{T}}=\left[\begin{array}{l}
x_{1} \\
x_{2} \\
1.4
\end{array}\right]
$$



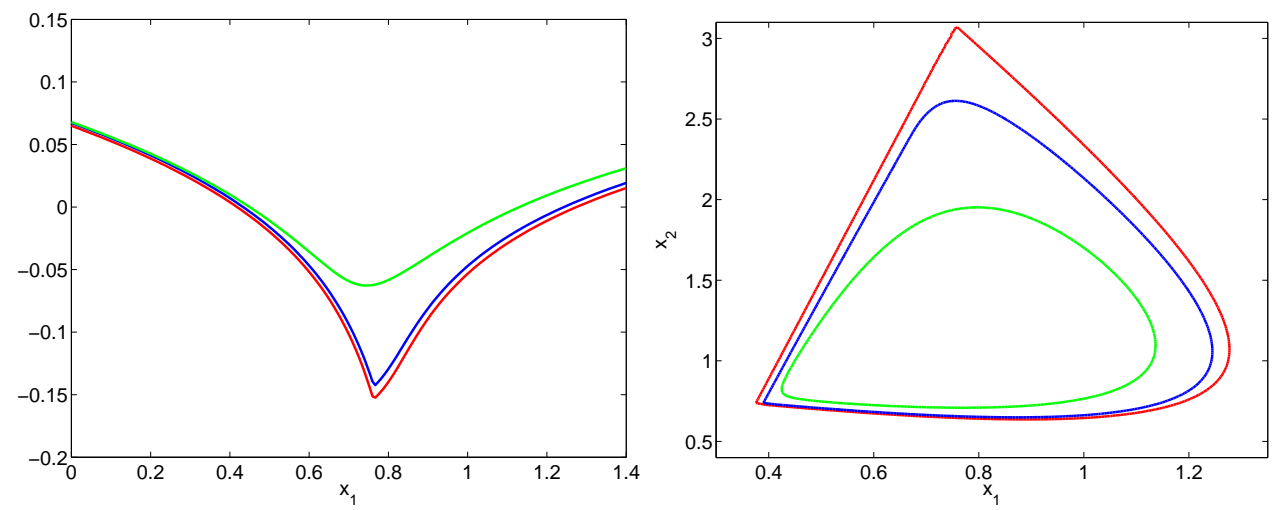

FIG. 6.1. Evolution w.r.t. $x_{1}$ (left) and stability regions (right) of the spectral abscissa $\alpha$ (in red), pseudo-spectral abscissa $\alpha_{\epsilon / 2}$ (in blue) and smoothed spectral abscissa $\tilde{\alpha}_{\epsilon}$ (in green) of the example in Subsection 6.1 with smoothing parameter $\epsilon=0.004$.

Figure 6.1 shows the $\epsilon$-smoothed spectral abscissa for $\epsilon=4 \cdot 10^{-3}$, in comparison with the pseudo-spectral abscissa (with an $\epsilon$ half as large) and the spectral abscissa $(\epsilon=0)$. In the left frame $x_{2}=1$ is held fixed. In the right frame both $x_{1}$ and $x_{2}$ are free and the contours of the stability regions, i.e. the regions where the respective measure is negative, are drawn. On both figures, we can clearly observe the smoothness of $\tilde{\alpha}_{\epsilon}$ (in green) in contrast with the nonsmooth behaviour of the spectral (in red) and pseudo-spectral (in blue) abscissa when the parameters are varied.

The ordering of $\tilde{\alpha}_{\epsilon}, \alpha_{\epsilon / 2}$ and $\alpha$ as stated by Theorems 2.6 and 5.1 is also confirmed. On the left, the curve of the smoothed spectral abscissa is everywhere above the two other curves, and on the right the $\tilde{\alpha}_{\epsilon}$-stability region is nicely contained within the stability regions of the pseudo-spectral and spectral abscissa.

6.2. Turbo generator model. Next, we treat Problem 'TG1' of Leibfritz's control problem database [18], which models a nuclear powered turbo generator. This system has also been used as an example in [11] for robust stability optimization using the pseudo-spectral abscissa in combination with the gradient sampling algorithm.

To analyze the influence of the smoothing parameter $\epsilon$ upon $\tilde{\alpha}_{\epsilon}$, we perform a standard BFGS minimization of the smoothed spectral abscissa for a set of $\epsilon$-values between 1 and $10^{-10}$, i.e. ranging from relatively large to very small.

Figure 6.2 shows the evolution of the minimized smoothed spectral abscissa for this $\epsilon$-range. It is verified that with $\epsilon$ becoming smaller the smoothed spectral abscissa decreases monotonically and the gap with the spectral abscissa narrows. Of course, as the smoothed spectral abscissa converges to the spectral abscissa for $\epsilon \rightarrow 0$, the optimization problems become more nonsmooth and thus harder. This is confirmed by Table 6.1, where next to the resulting minima for each $\epsilon$, the number of BFGSiterations (averaged out over 10 random starting points) needed to solve the respective optimization problems is listed. For small $\epsilon$, i.e. poor smoothing, this number becomes huge. But, having the smoothing parameter at hand to tune the smoothing, this problem can be somewhat circumvented by following a homotopy strategy. Iteratively decreasing $\epsilon$, and each time using the minimizer of the previous problem as initial point, we can decrease the amount of required iterations drastically. The number of iterations and the resulting minima for this strategy are listed in Table 6.1 next to the ones for which random starting points were used. 


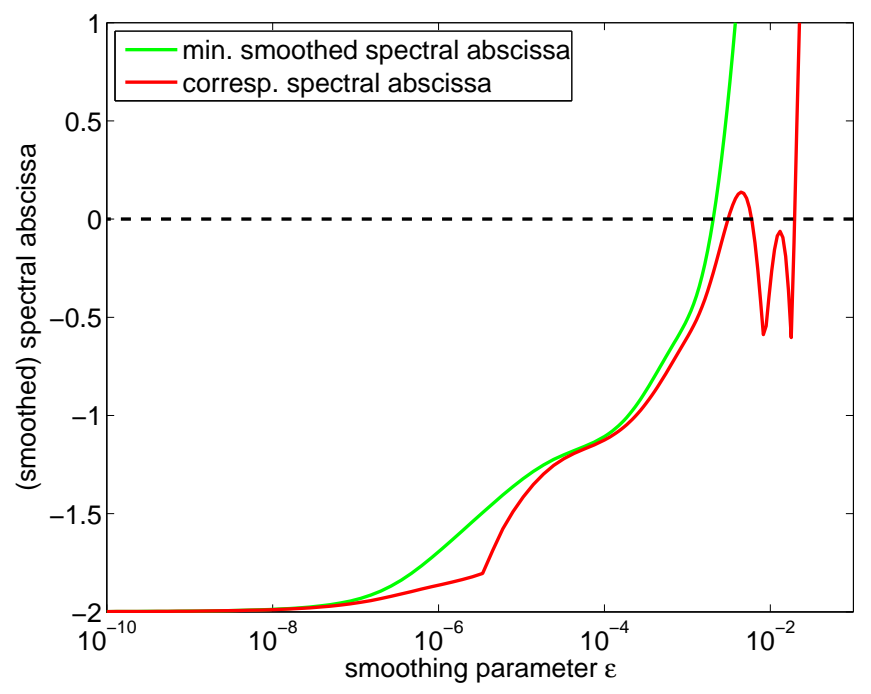

FIG. 6.2. The minimal smoothed spectral abscissa $\tilde{\alpha}_{\epsilon}$ for $\epsilon$ ranging from $10^{-10}$ to $10^{-1}$, compared to the corresponding spectral abscissa $\alpha$.

\begin{tabular}{crrrr}
\hline $\log _{10} \epsilon$ & \multicolumn{1}{c}{$\min _{x} \tilde{\alpha}_{\epsilon}(x)$} & \multicolumn{1}{l}{ iters } & \multicolumn{1}{c}{$\alpha_{\epsilon / 2}\left(x^{*}\right)$} & \multicolumn{1}{r}{$\alpha\left(x^{*}\right)$} \\
\hline 0 & $86.920558 / 86.920558$ & $35 / 32$ & 4.170208 & 9.899472 \\
-1 & $23.951317 / 23.951317$ & $37 / 33$ & 5.267947 & 5.447648 \\
-2 & $3.925292 / 3.925292$ & $29 / 29$ & -0.327800 & -0.272926 \\
-3 & $-0.508181 /-0.508181$ & $34 / 62$ & -0.600857 & -0.598826 \\
-4 & $-1.107119 /-1.107119$ & $69 / 54$ & -1.125056 & -1.124731 \\
-5 & $-1.328287 /-1.328287$ & $104 / 80$ & -1.427324 & -1.426787 \\
-6 & $-1.694445 /-1.694445$ & $102 / 65$ & -1.864124 & -1.864049 \\
-7 & $-1.938475 /-1.938475$ & $252 / 57$ & -1.955631 & -1.955624 \\
-8 & $-1.987303 /-1.987303$ & $292 / 125$ & -1.988801 & -1.988801 \\
-9 & $-1.996336 /-1.996246$ & $1522 / 51$ & -1.996542 & -1.996542 \\
-10 & $-1.998646 /-1.998587$ & $1827 / 47$ & -1.998680 & -1.998680 \\
\hline
\end{tabular}

TABLE 6.1

Solutions to the minimization of the smoothed spectral abscissa of the turbo generator model for designated $\epsilon$-values (without homotopy/with homotopy), and the corresponding pseudo-spectral and spectral abscissae.

Figure 6.3(a)-(d) plots the pseudospectra in the smoothed spectral abscissa minimizers for 4 selected smoothing levels, namely $\epsilon=2 \cdot 10^{-1,-3,-5,-7}$. For the first value of $\epsilon$, both the smoothed and spectral abscissa are positive and the minimizer is not stabilizing, as seen in the spectrum plotted in Figure 6.3(a). For $\epsilon=2 \cdot 10^{-3}$, the minimal smoothed spectral abscissa equals $-0.0270 \ldots$ and thus yields a stable system. In Figure 6.3(b), the eigenvalues are indeed all in the left half complex plane. Since the minimum is very close to zero, we can expect $2 \cdot 10^{-3}$ to be close to the maximal $\epsilon$ for which a stabilizing solution can be found. Solving optimization problem (4.3) yields an optimal value of $2.048 \cdot 10^{-3}$, which is indeed only slightly higher. Note that this optimum also corresponds to the $H_{2}$-norm minimizer, with an $H_{2}$-norm of approximately 22.1. Further decrease of $\epsilon$ results in smaller and smaller minimal smoothed spectral abscissae. As observed in Figure 6.3(c)-(d), the rightmost eigenvalues of the optimal spectra become more and more aligned on a vertical line, which resembles 


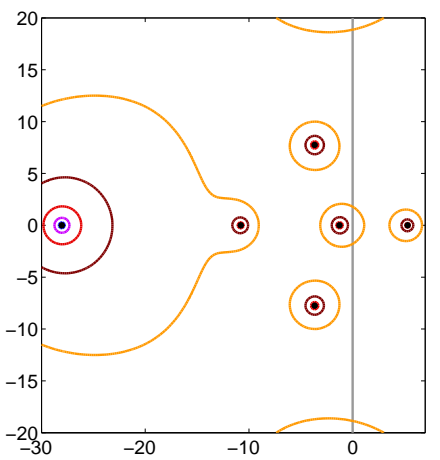

(a) $\epsilon=2 \cdot 10^{-1}$

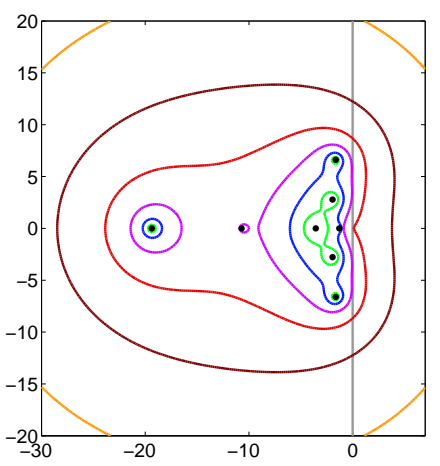

(c) $\epsilon=2 \cdot 10^{-5}$
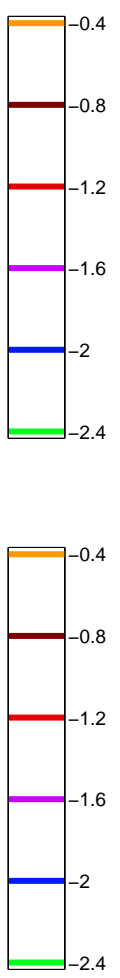

$-2.4$
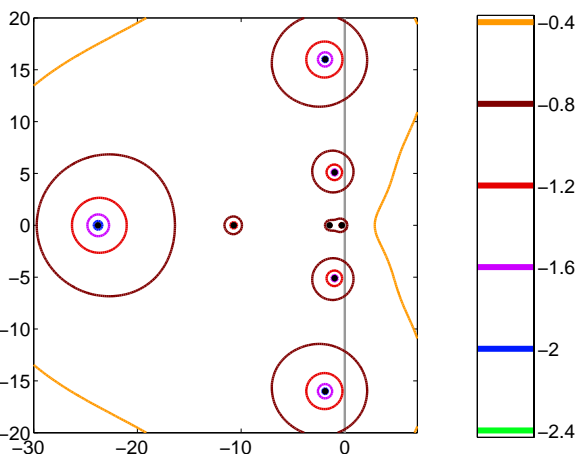

(b) $\epsilon=2 \cdot 10^{-3}$
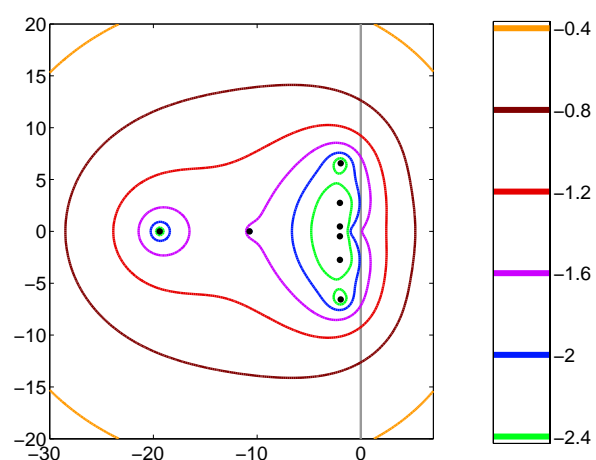

(d) $\epsilon=2 \cdot 10^{-7}$

FiG. 6.3. Pseudospectra of the turbo generator model in the minimizers of the smoothed spectral abscissa $\tilde{\alpha}_{\epsilon}(A(x))$ (problem (4.1)) for four $\epsilon$-values as used in Table 6.1.

the typical spectrum configuration for a minimized spectral abscissa. We thus have a range of stabilizing $\epsilon$ with solutions that compromise between the minimal spectral abscissa (fast response time) on the one hand, and the minimal $\mathrm{H}_{2}$-norm (robustness against noise) on the other hand. Remark that minimizing the pseudo-spectral abscissa delivers a similar robustness trade-off between the minimal spectral abscissa and the minimal $H_{\infty}$-norm.

If we have another look at the pseudospectra of Figure 6.3(b), (c) and (d), we see that their level sets with values $10^{-1.2}, 10^{-1.6}$ and $10^{-2}$ (in red, purple and blue respectively) are just contained in the left half complex plane, implying an increase of their $H_{\infty}$-norms when $\epsilon$ is increased. So, although the $H_{\infty}$-norm was not minimized here, the set of smoothed spectral abscissa minimizers appears to result in the same qualitative $H_{\infty}$ behaviour as would be the case for a range of pseudo-spectral abscissa minimizations.

To compare these two approaches, we define $x_{1}^{*}(s)$ and $x_{2}^{*}(s)$ as the respective minimizers in function of $s<0$ of the smoothed and pseudo-spectral abscissa, with smoothing epsilons $\epsilon_{1}(s)$ and $\epsilon_{2}(s)$, such that the attained minima equal $s$. Concisely,

$$
\begin{aligned}
& \tilde{\alpha}_{\epsilon_{1}(s)}\left(A\left(x_{1}^{*}(s)\right)\right)=\min _{x} \tilde{\alpha}_{\epsilon_{1}(s)}(A(x))=s, \\
& \alpha_{\epsilon_{2}(s)}\left(A\left(x_{2}^{*}(s)\right)\right)=\min _{x} \alpha_{\epsilon_{2}(s)}(A(x))=s .
\end{aligned}
$$




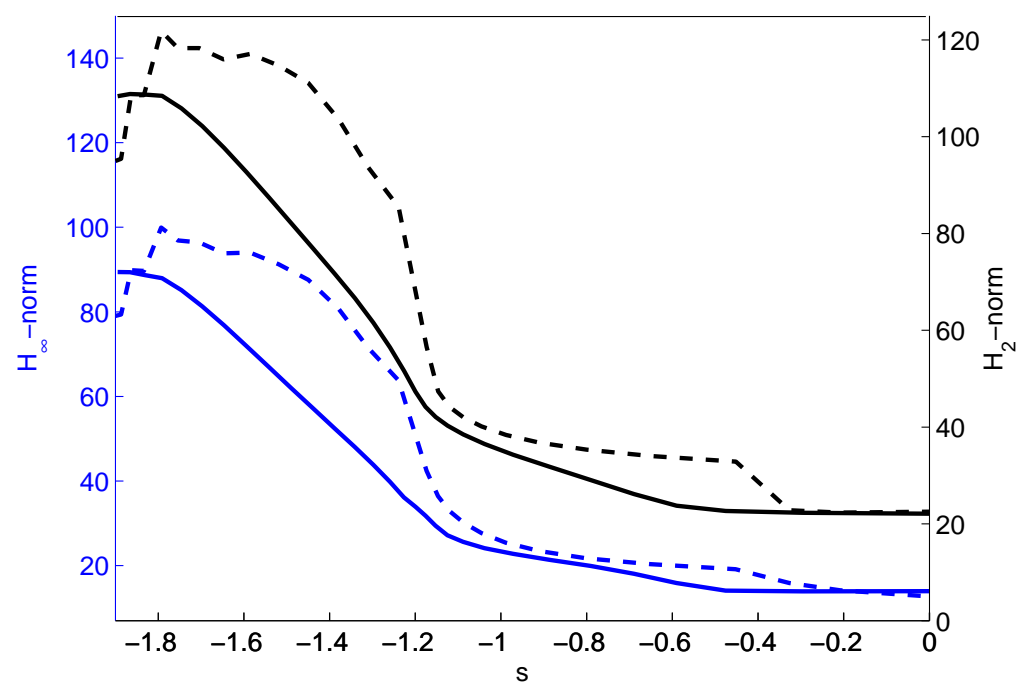

FIG. 6.4. $H_{2}$ - (in black) and $H_{\infty}$-norm (in blue) for the range of minimizers obtained by the minimization of the smoothed spectral abscissa (full lines) and of the pseudo-spectral abscissa (dashed lines).

According to the remark following Theorem 4.1, $x_{1}^{*}(s)$ and $x_{2}^{*}(s)$ minimize the $H_{2^{-}}$ norm and $H_{\infty}$-norm of a shifted system with transfer function $\mathbf{H}_{s}$.

In Figure 6.4, we show the values as a function of $s$ of the norms $\left\|z I-A\left(x_{1}^{*}(s)\right)\right\|_{\mathcal{H}_{2}}$ and $\left\|z I-A\left(x_{2}^{*}(s)\right)\right\|_{\mathcal{H}_{2}}$. In other words, we compare the $H_{2}$-norms of the unshifted transfer function, evaluated in the smoothed spectral abscissa minimizers $x_{1}^{*}$ on the one hand, and the pseudo-spectral abscissa minimizers $x_{2}^{*}$ on the other hand. We see that, except for $s$ very close to the minimal spectral abscissa, the $H_{2}$-norms of the smoothed spectral abscissa minimizers are smaller than those of the pseudo-spectral abscissa minimizers. For $s$ approaching zero, the difference between the two $H_{2}$-norms becomes very small. This implies that the optimal $H_{\infty}$-minimizer, i.e. the pseudospectral abscissa minimizer for which $s=0$, is accompanied by an $H_{2}$-norm that is only slightly suboptimal compared to the actual optimal $H_{2}$-norm.

We can make the same comparison for the $H_{\infty}$-norm. Also in Figure 6.4, we plotted $\left\|z I-A\left(x_{i}^{*}(s)\right)\right\|_{\mathcal{H}_{\infty}}$ for $i=1,2$. For $s=0$, the difference between the optimal $H_{\infty}$-norm and the suboptimal $H_{\infty}$-norm evaluated in the smoothed spectral abscissa minimizer is again very small. Surprisingly, for the other shifts, the $H_{\infty}$-norms of the smoothed spectral abscissa minimizers outperform the $H_{\infty}$-norms of the pseudospectral abscissa minimizers most of the time.

6.3. Computational cost. Finally, we compare the computational cost of the smoothed spectral abscissa $\tilde{\alpha}_{\epsilon}$ with two other robust stability measures: the pseudospectral abscissa $\alpha_{\epsilon}$ and the robust spectral abscissa $\alpha_{\delta}$. Each of these measures can be used as $\Phi_{\text {stab }}$ in (1.1) and as such, will be evaluated several times in the inner iterations of an optimization algorithm. Thus, the efficiency by which $\Phi_{\text {stab }}$ can be evaluated, has a direct influence on the overall efficiency of the optimization method for solving (1.1).

The details of the numerical methods used to compute each measure are listed in 


\begin{tabular}{|c|c|c|c|}
\hline$\Phi_{\text {stab }}$ & algorithm & convergence & inner solve (software) \\
\hline$\alpha_{\epsilon}$ & criss-cross $[10]$ & quadratic & Hamiltonian (Hapack based on [5]) \\
\hline$\alpha_{\delta}$ & bisection $[8]$ & linear & SDP (YALMIP [19], SeDuMi [24]) \\
\hline$\tilde{\alpha}_{\epsilon}$ & Dekker-Brent & superlinear & Lyapunov (Bartels-Stewart [4]) \\
\hline
\end{tabular}

Algorithms to compute the three stability measures.

\begin{tabular}{|c|c|c|c|c|c|}
\hline \multirow{2}{*}{ Ex. } & \multirow{2}{*}{$\Phi_{\text {stab }}$} & \multirow{2}{*}{$\begin{array}{c}\epsilon, \delta \\
(\text { logspace) }\end{array}$} & $\min$ & mean & $\max$ \\
\hline & & & $(\mathrm{sec})$ & $(\mathrm{sec}) \quad(\mathrm{its})$ & $(\mathrm{sec})$ \\
\hline \multirow{3}{*}{1} & $\alpha_{\epsilon}$ & $(-15,0,20)$ & $1.20 \mathrm{e}-03 \quad(3)$ & $1.79 \mathrm{e}-03$ & $2.71 \mathrm{e}-03$ \\
\hline & $\alpha_{\delta}$ & $(-2,0,20)$ & $5.58 \mathrm{e}+00(32)$ & $6.18 \mathrm{e}+00 \quad(32)$ & $7.71 \mathrm{e}+00$ \\
\hline & $\tilde{\alpha}_{\epsilon}$ & $(-15,0,20)$ & $4.23 \mathrm{e}-03(7)$ & $7.92 \mathrm{e}-03 \quad(16)$ & $1.57 \mathrm{e}-02$ \\
\hline \multirow{3}{*}{2} & $\alpha_{\epsilon}$ & $(-12,0,20)$ & $2.162 \mathrm{e}-03 \quad(3)$ & $3.094 \mathrm{e}-03 \quad(5)$ & $5.494 \mathrm{e}-03$ \\
\hline & $\alpha_{\delta}$ & $(-03,0,20)$ & $2.998 \mathrm{e}+01(54)$ & $1.798 \mathrm{e}+02(149)$ & $1.636 \mathrm{e}+03(\geq 999)$ \\
\hline & $\tilde{\alpha}_{\epsilon}$ & $(-15,0,20)$ & $6.312 \mathrm{e}-03(10)$ & $1.257 \mathrm{e}-02 \quad(22)$ & $2.204 \mathrm{e}-02$ \\
\hline
\end{tabular}

Timings and inner iterations of the three stability measures. The timings were done with 100 samples and the inner iterations are given as the number of inner solves in Table 6.3.

Table 6.3. From these, the criss-cross algorithm with a structure preserving Hamiltonian eigenvalue solver is to be preferred. To the best of our knowledge, the listed bisection algorithm is the only known implementation for computing $\alpha_{\delta}$. Specifically, we bisect until an absolute tolerance of $10 \epsilon_{\text {mach }}$ is satisfied and in each bisection step we check the feasibility of an SDP with SeDuMi 1.1R3.

Regarding the smoothed spectral abscissa, we use Dekker-Brent, implemented by MATLAB's fzero with an absolute tolerance $\epsilon_{\text {mach }}$, to find the unique root of the function $g(s):=1 / f(A, s)-\epsilon$. The reason for using the reciprocal instead of $f(A, s)-1 / \epsilon$ is that the former is better behaved numerically. Most of the time, we observed superlinear convergence. Recall that evaluating $f(A, s)$ involves solving a Lyapunov equation, which is done by the Bartels-Stewart algorithm, implemented by lyap in MATLAB.

We remark that our implementation for computing $\tilde{\alpha}_{\epsilon}$ is very preliminary, but it seems to work well for the model problems we tried. Besides some heuristics on setting up a bracketing interval, the procedure is quite robust. As far as efficiency goes, there is a lot of room for improvement. An obvious improvement is the inner loop of fsolve where $f(A, s)$ is evaluated for fixed $A$ but different shifts $s$. Since we solve the Lyapunov equations independently for each shift, we do not make use of the fact that we can reuse the computed Schur factorizations in Bartels-Stewart. In exact arithmetic, only one factorization would suffice. Furthermore, using DekkerBrent to solve $g(s)=0$ has the benefit of robustness, but we sometimes need a lot of work to find a bracketing interval. Since $f(A, s)$ is smooth and convex, a safe-guarded method based on Newton, may be more efficient. However, it is beyond the scope of the current article to implement all this.

In Table 6.3 we have summarized timings for the systems that we have examined earlier with the control parameters $x$ set to zero. However, since these three measures are quite different, comparing them is somewhat arbitrary. In order to have an impression of the computational cost, we computed each measure for a sensible range of its regularization parameter $\epsilon$ or $\delta$. It is clear from the table that the pseudo- 
and smoothed spectral abscissa are comparable in computational cost, and that the robust spectral abscissa is orders of magnitudes slower.

7. Conclusions. A smooth relaxation of the nonsmooth spectral abscissa function was introduced as an alternative stability measure, with the advantage that derivative based optimization techniques can readily be used for its optimization. Formulae regarding the efficient computation and derivative evaluation of the smoothed spectral abscissa were deduced based on the solution of a primal-dual Lyapunov equation pair.

Besides its direct minimization, which can be used to find stabilizing controllers, a second optimization formulation was shown to be applicable to solve fixed-order $\mathrm{H}_{2}$ optimization problems. Moreover, a guaranteed bound on the distance to instability was established by relating the results to the $H_{\infty}$-norm. The robust stabilization by use of these two optimization problems involving the smoothed spectral abscissa was illustrated with numerical examples and also a comparative study of the computational complexity cost was made.

\section{REFERENCES}

[1] P. Apkarian And D. Noll, Nonsmooth H-infinity synthesis, IEEE Transactions on Automatic Control, 51 (2006), pp. 71-86.

[2] - Nonsmooth optimization for multidisk H-infinity synthesis, European Journal of Control, 12 (2006), pp. 229-244.

[3] V. Balakrishnan and L. Vandenberghe, Semidefinite programming duality and linear timeinvariant systems, IEEE Transactions on Automatic Control, AC-48 (2003), pp. 30-41.

[4] R. H. Bartels and G. W. Stewart, Solution of the matrix equation $A X+X B=C$, Communications of the ACM, 15 (1972), pp. 820-826.

[5] P. Benner, V. Mehrmann, and H. Xu, A numerically stable, structure preserving method for computing the eigenvalues of real Hamiltonian or symplectic pencils, Numer. Math., 78 (1998), pp. 329-358.

[6] R. P. BREnt, Algorithms for Minimization without Derivatives, Prentice-Hall, Englewood Cliffs, NJ, 1973.

[7] J. V. Burke, D. Henrion, A. S. Lewis, and M. L. Overton, HiFOO - a matlab package for fixed-order controller design and H-infinity optimization, in Proceedings of the 5th IFAC Symposium on Robust Control Design, Toulouse, France, 2006.

[8] J. V. Burke, A. S. Lewis, And M. L. Overton, Two numerical methods for optimizing matrix stability, Linear Algebra and its Applications, 351 (2002), pp. 147-184.

[9] - Optimization and pseudospectra, with applications to robust stability, SIAM J. Matrix Anal. Appl., 25 (2003), pp. 80-104.

[10] - Robust stability and a criss-cross algorithm for pseudospectra, IMA J. Numer. Anal., 23 (2003), pp. 359-375.

[11] - A nonsmooth, nonconvex optimization approach to robust stabilization by static output feedback and low-order controllers, in Proceedings of 4th IFAC Symposium on Robust Control Design, Milan, Italy, 2004, pp. 175-181.

[12] - A robust gradient sampling algorithm for nonsmooth, nonconvex optimization, SIAM J. Optimization, 15 (2005), pp. 751-779.

[13] J. V. Burke And M. L. Overton, Differential properties of the spectral abscissa and the spectral radius for analytic matrix-valued mappings, Nonlinear Analysis: Theory, Methods \& Applications, 23 (1994), pp. 467-488.

[14] - Variational analysis of non-Lipschitz spectral functions, Math. Programming, 90 (2001), pp. $317-352$.

[15] M. Diehl, K. Mombaur, And D. Noll, Stability optimization of hybrid periodic systems via a smooth criterion, Tech. Rep. 07-97, ESAT-SISTA, K.U.Leuven, Belgium, 2007.

[16] A. Graham, Kronecker Products and Matrix Calculus With Applications, Halsted Press, John Wiley and Sons, New York, 1981.

[17] P. LANCASTER, Explicit solutions of linear matrix equations, SIREV, 12 (1970), pp. 544-566.

[18] F. Leibfritz, COMPleib: COnstraint Matrix-optimization Problem library - a collection of 
test examples for nonlinear semidefinite programs, control system design and related problems, tech. rep., Universität Trier, 2004.

[19] J. LÖFBerg, YALMIP : A toolbox for modeling and optimization in MATLAB, in Proceedings of the CACSD Conference, Taipei, Taiwan, 2004

[20] M. Mammadov and R. ORsi, H-infinity synthesis via a nonsmooth, nonconvex optimization approach, Pacific Journal of Optimization, 1 (2005), pp. 405-420.

[21] W. Michiels And D. Roose, An eigenvalue based approach for the robust stabilization of linear time-delay systems, International Journal of Control, 76 (2003), pp. 678-686.

[22] D. Noll And P. ApkARIAn, Spectral bundle methods for nonconvex maximum eigenvalue functions. Part 1: first-order methods, Math. Programming Series B, 104 (2005), pp. 701-727.

[23] - Spectral bundle methods for nonconvex maximum eigenvalue functions. Part 2: secondorder methods, Math. Programming Series B, 104 (2005), pp. 729-747.

[24] J. F. Sturm, Using SeDuMi 1.02, a MATLAB toolbox for optimization over symmetric cones, Optim. Methods Softw., 11-12 (1999), pp. 625-653.

[25] N. Trefethen And M. Embree, Spectra and Pseudospectra - The Behavior of Nonnormal Matrices, Princeton University Press, NJ, 2005.

[26] J. Vanbiervliet, K. Verheyden, W. Michiels, and S. Vandewalle, A nonmooth optimisation approach for the stabilisation of time-delay systems, ESAIM: Control, Optimisation and Calculus of Variations, (2007). To appear.

[27] K. Zhou, J. C. Doyle, And K. Glover, Robust and Optimal Control, Prentice Hall, NJ, 1996. 